\title{
Design of Grazing Incidence Monochromators Involving Unconven:ional Gratings
}

\author{
Wayne R. McKinney \\ Accelerator and Fusion Research Division \\ Lawrence Berkeley Labontory \\ One Cyclotron Roac \\ Berkeley, CA 94720
}

\author{
Christopher Palmer \\ Milton Roy Company \\ Analytical Systems Division \\ 820 Linden Avenue \\ Rochester, NY 14625
}

Presented at SPIE's Symposium on Lasers and Optics

15-20 January, 1989

Los Angeles, CA USA

To be Published in SPIE Proceedings 1055

This work was supported by the Direclor, Office of Energy Research, Office of Basic Energy Sciences, Materials Sciences Division of the U. S. Department of Energy under Contract No. DE-AC03-76SF00098 


\title{
DESIGN OF GRAZING INCIDENCE MONOCHROMATORS INVOLVING UNCONVENTIONAL GRATINGS
}

\author{
Wayne R. McKinney \\ Accelerator and Fusion Research Division \\ Center for X-Ray Optics \\ Lawrence Berkeley Laboratory \\ 1 Cyclotron Rd. \\ Berkeley, CA 94720 \\ Christopher Palmer \\ Milion Roy Company \\ Analytial Systems Division \\ 820 Linden Ave. \\ Rochester, NY. 14625
}

\section{Introduction}

The manufacture of varied line space (VLS) gratings was pioneered by Handa and co-workers of Japan, 1 and Gerasimov and co-workers in the Soviet Union. ${ }^{2}$ Ruling engines were modified to allow the groove spacing to vary in a continuous manner, the grooves often remaining straight and parallel. This type of grating can also be obtained in the United States from Perkin Elmer Co. of Irvine, CA. Other VLS gratings involving tan shaped or concentric grooves have been developed by these groups and Hyperfine, Inc. of Boulder, CO. Monochromators using VLS for the UV and higher energy regions have been developed by Hettrick. ${ }^{3}$ We review the basic aspects of second order focusing of straight and peraltel grooved varied line space gratings in both converging and collimaved light in a more explicit and detailed manner that we have found in the literature. The effects of the VLS correction to the location of the focal curve for grazing incidence geometries are found to be very significant.

\section{Geometry}

We consider two cases shown in Fig. la and $1 \mathrm{~b}$. The light can be converging to a focal point behind a VLS grating as in Fig. la, or impinging onto the grating as a parallel beam as in Fig. lb. The grating is assumed to be a plane or have a relatively long radius of curvature.
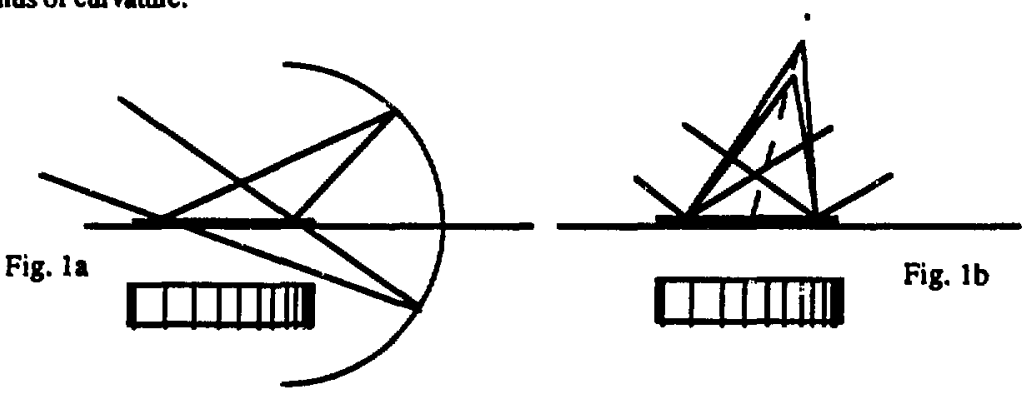

We adopt the familiar coordinate system and optical path function analysis of Noda, et al. ${ }^{4}$ which is shown in Fig. 2. The path function is written as the difference between the palh taken by a general ray and the pole ray plus a term which accourils

$$
\Psi=\langle A P B\rangle-\angle A O B\rangle+\mathrm{Nm} \lambda
$$

for the presence of the grating grooves. We then choose to expand the groove spacing about the spacing at the center of the grating, do. 


$$
d=d_{0}\left[1+\mu y+v y^{2}+x y^{3}+\ldots\right]
$$



Fig. 2

Here d, the groove spacing as a function of distance along the grating width $y$, is equal to the partial derivative of $y$ with respect to the groove number $N$. The defocus coefficient. $F_{20}$, can now be found by taking the second derivative of $\Psi$ with respect to $y$, and evaluating at $y=z=m$

$$
\begin{aligned}
& \left.\frac{\partial^{2} \Psi}{\partial y^{2} 0}=F_{D}=M_{z}+\frac{\partial^{2} N}{\partial y_{0}}, \therefore \quad=M_{D}+\left[\frac{\delta}{\delta y}\left(\frac{\delta N}{\delta y}\right)\right]_{0} m \lambda=M_{D}+\left[\frac{\delta}{\delta y}\left(\frac{1}{d}\right)\right]\right]_{0} m \lambda \\
& \left.=M_{D}+\left[\frac{\delta}{\delta y}\left(\frac{\delta N}{\delta y}\right)\right]\right]_{0} \lambda=M_{D}+\left[\frac{\delta}{\delta y}\left(\frac{1}{d}\right)\right]_{0} m \lambda \quad=M_{D}+\left[\frac{\delta}{\delta y}\left(\frac{1}{d\left(1+\mu y+v y^{2}+k y^{3}+\ldots\right)}\right)\right]_{0} m \lambda \\
& \Rightarrow F_{D}=M_{D}-\frac{\mu m \lambda}{d_{0}}=M_{D}-\mu(\sin \alpha+\sin \beta)
\end{aligned}
$$

2.3

We have used the grating equation to arrive at the last equation. $\mathrm{M}_{20}$ represents the classical terms which are not associated with the VLS correction. Inserting these, where a20 depends on the shape of the grating, gives.

$$
F_{20}=\frac{\cos ^{2} \alpha}{r}+\frac{\cos ^{2} \beta}{r}-2 a_{20}(\cos \alpha+\cos \beta)-\mu(\sin \alpha+\sin \beta)
$$

We include without derivation the equivalent expressions for the coma and spherical aberration coefficients.

$$
\begin{aligned}
F_{30}=M_{30} & \left.+\frac{2 m \lambda}{d_{0}}\left(\mu^{2}-v\right)=M_{00}+2\left(\mu^{2}-v\right)(\sin \alpha+\sin \beta)\right) \\
F_{00} & =M_{0}-\frac{6 m \lambda\left(\mu^{3}-2 \mu v+\kappa\right)}{d_{0}} \\
& \left.=M_{00}-6\left(\mu^{3}-2 \mu v+x\right)(\sin \alpha+\sin \beta)\right)
\end{aligned}
$$

\section{Standard Case}

Our test case for evaluating the conditions of defocus is the following:

fixed deviation monochromator, half deviation angle $=87$ degrees

order of diffraction $=+1$ (inner order, $|\beta|<\alpha$ )

wavelength range $=20 \mathrm{~A}$ to $40 \mathrm{~A}$

focus of the converging ircident light 1 meter behind the grating

grooves per $\mathrm{mm}=1200$ at the cenicr of the grating

Following Hetrick, ${ }^{5}$ we set $F_{20}=0$ at the two extrema of the wavelength range, $20 \mathrm{~A}$ and $40 \mathrm{~A}$. These two equations can be solved for the ratio of the distance to horizontal focus from the grating pole ri' to the distance to the object point from the grating pole $r_{H}$, where we define $r_{H}$ as positive behind the graling, and insert a minus sign into each equation to compensate. 


$$
\frac{r_{\dot{H}}}{r_{H}}=\frac{\lambda_{1} \cos ^{2} \beta_{2}-\lambda_{2} \cos ^{2} \beta_{1}}{\lambda_{1} \cos ^{2} \alpha_{2}-\lambda_{2} \cos ^{2} \alpha_{1}}
$$

For our case $r_{H}^{1} / r_{H}=1.00448$. Either of the individual equations can be solved for $\mu$ to give $\mu=+1.986225$. This effectively puts the two available zeros of the defocus coefficient at the two chosen wavelengths. This coefficient is shown in Fig. 3.

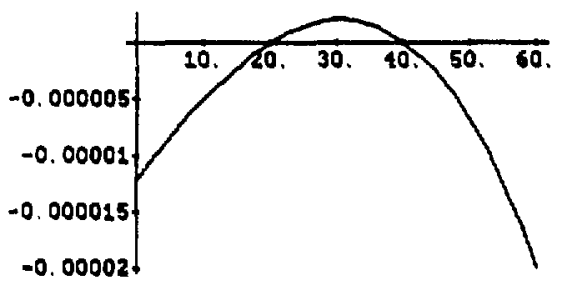

Fig. 3

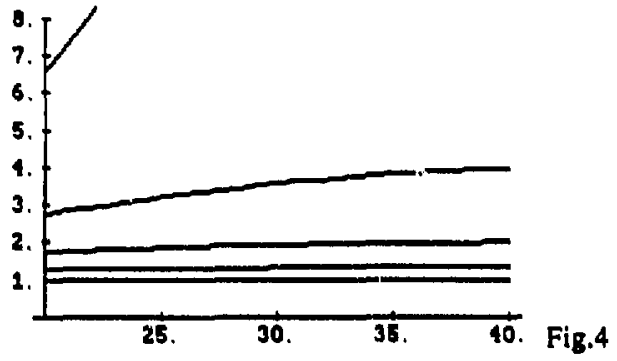

The effect of this remarkably low defocus is shown in Fig. 4. Here the value of ry'/ry is plotted for zero, 25, 50, 75 and $100 \%$ of $\mu=+1.986225$. We see that the distance 10 horizontal focus varies greatly for the $\mu=0$ case (no VLS). For full correction, the distance to focus is essentially flat on any scale that shows the case with $\mu=0$. If we define a "variance" for $\mathrm{rH}^{\prime}$ as a quantitative measure of its deviation from perfect focal correction, and rationalize to the lowest value to guananiee that we will count only "Rauness"; then the correction is seen to be quite dramatic in Fig. 5. The log 10 of the "variance" or merit function $M$, defined below, is plotted as a function of $\mu$.

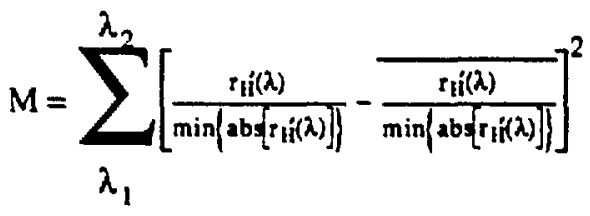

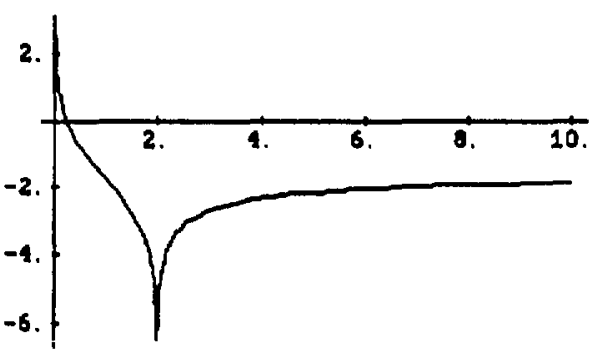

Fig. 5

Since in Fig. 4 we plot $\mathrm{rH}_{\mathrm{T}} / \mathrm{H}$ with $\mathrm{r}$ at the standard value of 1 , we can see that at least for our standard case the optimal VLS correction to this order occurs maturally near the point where the light woukd focus without the grating. This is very fortunate, since in the sagittl direction the straight grooved grating does not affect the focusing at all. We have varied the parameters of the standard case and find that the best flatening of the horizontal focal curve does in general occur near unity magnification for the grating. This does not occur when the VLS grating is used to focus parallel light. Leuing $\mathrm{rH}$-.>0 in equation $2.4\left(\mathrm{a}_{20}-0\right)$ and solving for $\mathrm{rH}^{\prime}$ gives,

$$
r_{H}=\frac{d \cos ^{2}(\beta)}{\mu m \lambda}
$$

showing that $\mu$ is just a scaling factor which detcrmines the distance to the tangential focus. The shape of the focal curve does not change as does the case of converging light. Changing $\mu$ moves the curve in and out, but does not "flatten" the curve, which is shown in Fig. 6 which plots $\mathrm{rH}^{1} / \mathrm{rH}_{\mathrm{H}}$ as a function of wavelength for $\mathrm{rH}_{\mathrm{H}}=1$. 


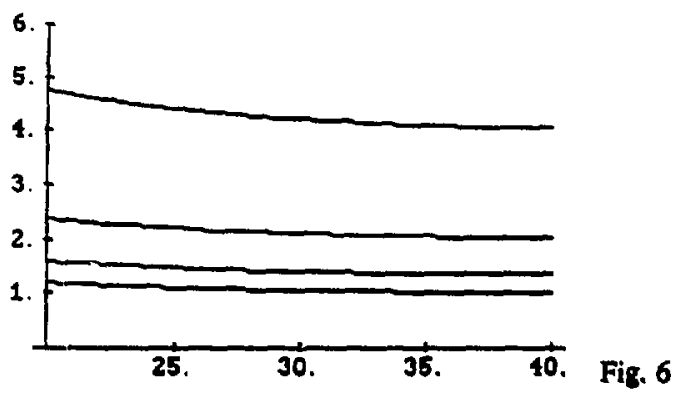

We see that the focal curve correction is not nearly as good as when the VLS grating is used in converging light. However for moderave resolution insuruments for synchrotron radiation beam lines where the radiation can have very low divergence, the VLS grating in collimald light can be used to edventuge. If the grating can be translated as it is rouled, as has been reported by ltou at $2 .{ }^{6}$, the small defect of focus can be compensated.

\section{Summary.}

We have shown the following:

1. Plane VLS graings provide profound focal curve correction when used at grazing incidence and in converging light.

2. Moderate resolving power instruments are possible using collimated light.

3. Optimal VLS linear spacing variations naturally give a tangential focal distance near the sagiltal focus for cases similar to these in converging light.

This work was supported in part by the Director, Office of Energy Research, Office of Basic Energy Sciences, Materials Sciences Division, of the U.S. Department of Energy under contract No. DE-AC03-76SF00098.

I T Harada and T. Kila, "Mechanically Ruled Aberration-Corrected Concave Gratings," Applied Opiics 19, 3987 (1980).

2 F. M. Gerasimov, E. A. Yaskovlev, I. V. Peisakhson, and B. V. Koshelev, "Concave Diffraction Gratings with Variable Spacing." Opt. Spektrosk. 28, 423 (1970)

3 Michael C. Hettrick, "Varied line space graings: past present and future," SPIE Proceedings 560, 96 (1985).

4 H. Noda. T. Namioka, and M. Seya, "Geometric Theory of the Grating," J. Opl., Soc. Am. 64, 1031 (1974).

5 Michael C Hetrick, James H. Underwood, Philip J. Bason, and Mark J. Eckart, "Resolving power of 35,000 (5 mA) in the extreme ultraviolet employine a grazing incidence spectrometer," Applied Oprics 27, 200 (1988).

6 Masaaki Itou. Tatswo Harada, and Toshiaki Kith, "Soft x-ray monochromator with a varied-space plane grating for synchrouron radiation: design and evaluation," Applied Optics, 28, 146 (1989).

\section{DISCLAIMPR}

This report was prepared as an account of work sponsored by an azency of the United States Government. Neither the United States Government nor any agency thereof, nor any of their employees, makes any warranty, express or implied, or assumes any legal liability or responsibility for the accuracy, completeness, or usefulness of any information, apperatus, product, or process disclosed, or represents that its use would not infringe privately owned rights. Reference herein to any specific commercial product, process, or service by trade nume, trademark, manufacturer, or otherwise does not necessarily constitute or imply its endorsement, recommendation, or favoring by the United States Government or any agency thereof. The views and opinions of authors expressed herein do not necessarily slate of reflect those of the United States Government or any agency thereof. 